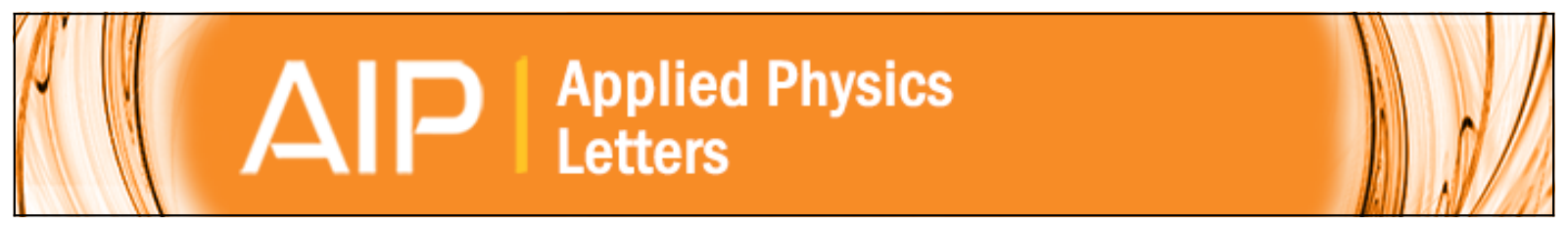

\title{
Isothermal tuning of exchange bias using pulsed fields
}

J. Nogués, J. Sort, S. Suriñach, J. S. Muñoz, M. D. Baró, J. F. Bobo, U. Lüders, E. Haanappel, M. R.

Fitzsimmons, A. Hoffmann, and J. W. Cai

Citation: Applied Physics Letters 82, 3044 (2003); doi: 10.1063/1.1565711

View online: http://dx.doi.org/10.1063/1.1565711

View Table of Contents: http://scitation.aip.org/content/aip/journal/apl/82/18?ver=pdfcov

Published by the AIP Publishing

\section{AlP Re-register for Table of Content Alerts}

\section{Create a profile. \\ Sign up today!}




\title{
Isothermal tuning of exchange bias using pulsed fields
}

\author{
J. Nogués ${ }^{a)}$ \\ Institució Catalana de Recerca i Estudis Avançats (ICREA) and Departament de Física, Universitat \\ Autònoma de Barcelona, 08193 Bellaterra, Spain \\ J. Sort, S. Suriñach, J. S. Muñoz, and M. D. Baró \\ Departament de Física, Universitat Autònoma de Barcelona, 08193 Bellaterra, Spain \\ J. F. Bobo and U. Lüders \\ LPMC, UMR 5830 CNRS-INSA-UPS, 31077 Toulouse, France \\ E. Haanappel \\ Laboratoire National des Champs Magnétiques Pulsés, 31432 Toulouse, France \\ M. R. Fitzsimmons \\ Los Alamos National Laboratory, Los Alamos, New Mexico 87545 \\ A. Hoffmann \\ Materials Science Division, Argonne National Laboratory, Argonne, Illinois 60439 \\ J. W. Cai \\ Institute of Physics, Chinese Academy of Sciences, 100080 Beijing, China
}

(Received 19 November 2002; accepted 10 February 2003)

\begin{abstract}
Exchange bias, $H_{E}$, and coercivity, $H_{C}$, of antiferromagnetic (AFM)/ferromagnetic bilayers can be adjusted, after deposition, at temperatures below the Néel temperature of the AFM by subjecting the samples to large pulsed fields (in excess of $H_{\text {Pulse }}=550 \mathrm{kOe}$ ). The efficiency of the process depends on the AFM system and the direction of the applied field with respect of the unidirectional anisotropy direction. Textured (111) $\mathrm{Fe}_{19} \mathrm{Ni}_{81} / \mathrm{Fe}_{50} \mathrm{Mn}_{50}$ bilayers show an $H_{E}$ reduction and a $H_{C}$ increase when the pulse field is applied antiparallel to the unidirectional anisotropy, while they only exhibit a reduction in $H_{C}$ when the pulse is applied parallel to their unidirectional anisotropy. On the other hand, textured (111) $\mathrm{NiO} / \mathrm{Co}$ bilayers exhibit a change of the angular dependence of $H_{E}$ when the pulse is applied away from the unidirectional anisotropy. The effects could be caused by field induced changes in the domain structure of the AFM or transitions in the AFM (spin-flop or AFM-paramagnetic). () 2003 American Institute of Physics. [DOI: 10.1063/1.1565711]
\end{abstract}

Exchange bias, i.e., the shift of the hysteresis loop along the field axis, ${ }^{1}$ resulting from the exchange coupling at the interface between ferromagnetic (FM) and antiferromagnetic (AFM) materials, plays a fundamental role in magnetoelectronic devices. ${ }^{2}$ To induce exchange bias, AFM-FM systems are usually either (i) field cooled through the Néel temperature, $T_{N}$, of the AFM or (ii) deposited in the presence of a field. ${ }^{1}$ At a fixed temperature, the loop shift, $H_{E}$, and the coercivity, $H_{C}$, of the system are controlled by intrinsic parameters such as AFM-FM coupling at the interface, FM and AFM thicknesses, interface roughness, or grain size. ${ }^{1}$ Hence, $H_{E}$ and $H_{C}$ should remain fixed after deposition.

However, it has been shown that extrinsic parameters, such as annealing, different cooling procedures, or ion irradiation can tune the values of $H_{E}$ and $H_{C}$ after sample growth. ${ }^{3-9}$ Irreversible approaches, such as high temperature annealing ${ }^{3}$ or ion irradiation ${ }^{4}$ induce structural changes in the bilayers. Hence, $H_{E}$ and $H_{C}$ can only be adjusted a limited number of times. Among the reversible techniques, cooling through $T_{N}$ in large fields, ${ }^{5-7}$ cooling in combinations of continuous and alternating fields, ${ }^{8}$ or cooling in zero field from different magnetization states, ${ }^{9}$ all require warming the bilayer above $T_{N}$. Another approach to tune $H_{E}$ and $H_{C}$ is to go to large negative fields to saturate the FM and to wait a

a)Electronic mail: josep.nogues@uab.es certain time and subsequently carry out the remaining part of the loop. ${ }^{10} H_{E}$ and $H_{C}$ depend on the waiting time. This procedure can be slow, thus often to enhance its efficiency the samples are warmed close to $T_{N}$. Warming the bilayers with AFMs with $T_{N} \gg 300 \mathrm{~K}$ could induce unwanted structural changes (e.g., interdiffusion) in the system, which could deteriorate the performance of the device. ${ }^{3}$ Moreover, in other AFM materials, such as $\mathrm{LaFeO}_{3}$ (Ref. 11) or $\alpha-\mathrm{Fe}_{2} \mathrm{O}_{3},{ }^{12}$ the samples cannot be warmed close or above $T_{N}$, either because the AFM decomposes (e.g., $\mathrm{LaFeO}_{3}$ ) or due to the exceedingly large $T_{N}$.

In this letter, we present a reversible process to tune $H_{E}$ and $H_{C}$ in exchange biased bilayers, with $T_{N}>300 \mathrm{~K}$, without the need to warm the samples above $T_{N}$. The procedure involves applying large field pulses at room temperature (RT). The field induced changes depend on the direction of the applied pulse and the type of AFM material.

Textured (111) $\quad \mathrm{Fe}_{19} \mathrm{Ni}_{81} / \mathrm{Fe}_{50} \mathrm{Mn}_{50}$ and textured (111) $\mathrm{NiO} / \mathrm{Co}$ were studied. The $\mathrm{Fe}_{19} \mathrm{Ni}_{81}(10 \mathrm{~nm}) /$ $\mathrm{Fe}_{50} \mathrm{Mn}_{50}(15 \mathrm{~nm})(\mathrm{FeNi} / \mathrm{FeMn})$ bilayer was sputtered at $\mathrm{RT}$ onto Corning glass. Nonmagnetic $\left(\mathrm{Ni}_{81} \mathrm{Fe}_{19}\right)_{50} \mathrm{Cr}_{50}$ was used as buffer and capping layers. A field of $H=400$ Oe was applied during growth to induce a unidirectional anisotropy. The $\mathrm{NiO}(20 \mathrm{~nm}) / \mathrm{Co}(15 \mathrm{~nm})$ samples were evaporated onto oxidized $\mathrm{Si}(100)$ substrates. The $\mathrm{NiO}$ layer was $e$-beam evaporated from a $\mathrm{Ni}$ ingot in an $\mathrm{O}_{2}$ atmosphere while the 


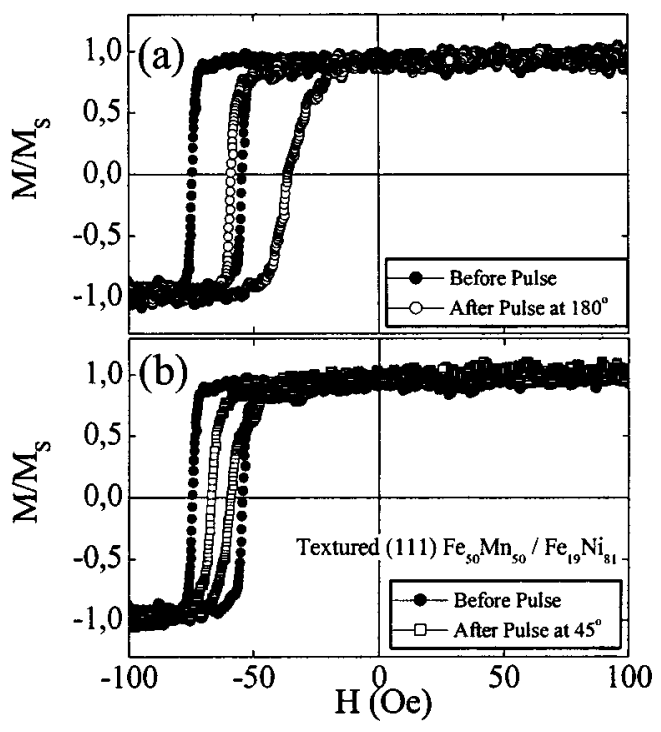

FIG. 1. Hysteresis loops measured along the unideirectional anisotropy direction for a $\mathrm{Fe}_{19} \mathrm{Ni}_{81} / \mathrm{Fe}_{50} \mathrm{Mn}_{50}$ bilayer (a) before (@) and after a field pulse at $180^{\circ}$ away from the unidirectional anistropy $(\bigcirc)$ and $(b)$ before $(\bullet)$ and after a field pulse at $45^{\circ}$ away from the unidirectional anistropy $(\square)$.

substrate was at $T_{S}=200^{\circ} \mathrm{C}$. Before the Co deposition the $\mathrm{O}_{2}$ was evacuated to $<2 \times 10^{-7}$ Torr. The Co layer was $e$-beam evaporated at $T_{S}=200^{\circ} \mathrm{C}$. The samples were capped by an Al layer.

The in-plane dependence of the hysteresis loops was measured at RT using a magneto-optic Kerr effect apparatus, before and after the application of field pulses. The pulsed field was always applied in the sample plane and along different directions with respect to the unidirectional anisotropy direction (UAD). The FeNi/FeMn bilayer was cut in several pieces and pulses were applied at $0^{\circ}, 45^{\circ}, 90^{\circ}$, and $180^{\circ}$ of the direction of the field applied during growth (i.e., the $\mathrm{UAD}$ ). For the $\mathrm{NiO} / \mathrm{Co}$ samples, the pulse was applied at $45^{\circ}$ of the direction of maximum $H_{E}$. The rise and fall times of the pulse are about 22 and $150 \mathrm{~ms}$, respectively, while the maximum achieved field is $H=556 \mathrm{kOe}$.

Shown in Fig. 1(a) are the hysteresis loops of the $\mathrm{FeNi} / \mathrm{FeMn}$ bilayer before and after applying a field pulse antiparallel to the UAD. There is a clear reduction of $H_{E}$ and an increase of $H_{C}$, after the $180^{\circ}$ field pulse. However, applying a pulse along $45^{\circ}$ [Fig. 1(b)] only results in the reduction of $H_{C}$. Moreover, the hysteresis loops become more asymmetric after the field pulses. When comparing the angular dependence of $H_{E}$ and $H_{C}$ for the FeNi/FeMn bilayer before and after field pulses applied along different directions (Fig. 2), one can observe that the shape of the angular dependence does not change significantly. However, $H_{E}$ becomes smaller as the pulse direction moves away from the UAD. Remarkably, the coercivity decreases, along the unidirectional axis, for pulses applied at $0^{\circ}$ (not shown), $45^{\circ}$, and $90^{\circ}$, while it increases considerably for pulses applied along $180^{\circ}$.

The NiO/Co bilayer exhibits a different behavior (Fig. 3). Although, $H_{E}$ decreases and $H_{C}$ increases after a $45^{\circ}$ field pulse, the angular dependence of $H_{E}$ changes considerably. Namely, a new UAD, i.e., the direction with maximum $H_{E}$, is created in a direction closer to the pulsed field.

From the results certain trends can be extracted: (i) the

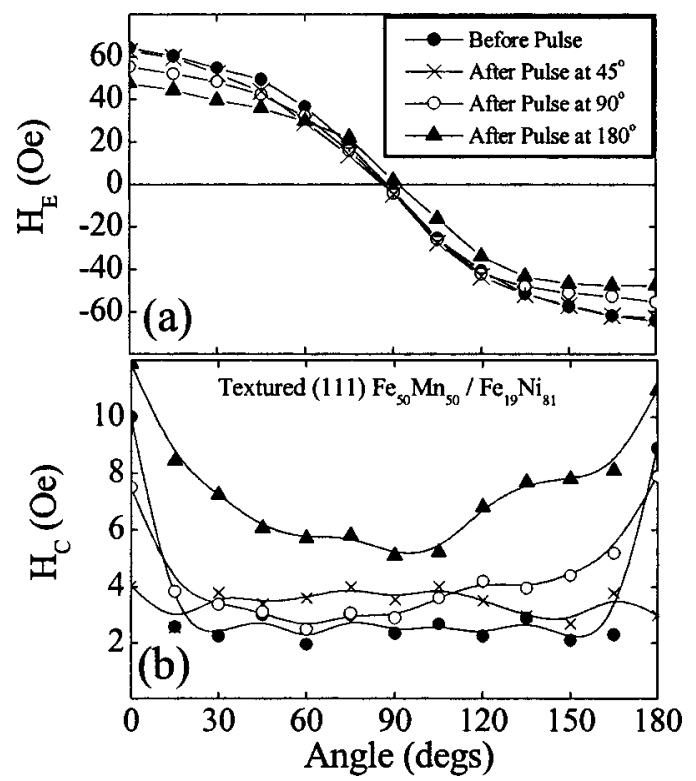

FIG. 2. Angular dependence of the exchange bias, $H_{E}$ (a), and coercivity, $H_{C}$ (b), for a $\mathrm{Fe}_{19} \mathrm{Ni}_{81} / \mathrm{Fe}_{50} \mathrm{Mn}_{50}$ bilayer before $(\bullet)$ and after the application of pulses along $45^{\circ}(\times), 90^{\circ}(\bigcirc)$, and $180^{\circ}(\mathbf{\Lambda})$ with respect to the unidirectional anisotropy. Lines are guides for the eye.

field induced changes appear to be maximum for pulses applied antiparallel to the UAD; (ii) the kind of tuning can be controlled by the pulse angle; and (iii) different AFMs with similar microstructure respond differently to the applied pulses.

The described field induced changes could have different origins, such as changes in the AFM domain structure or field induced transitions in the AFM. Several theories outline the importance AFM domains may have in exchange bias. ${ }^{13}$ Such AFM domains could be in metastable states. For example, changes in the AFM domain configuration have been claimed to be responsible for time dependence effects ${ }^{10}$ or training effects. ${ }^{14}$ Thus, such domains could probably be altered by the large pulsed fields. Certainly, in NiO single crys-

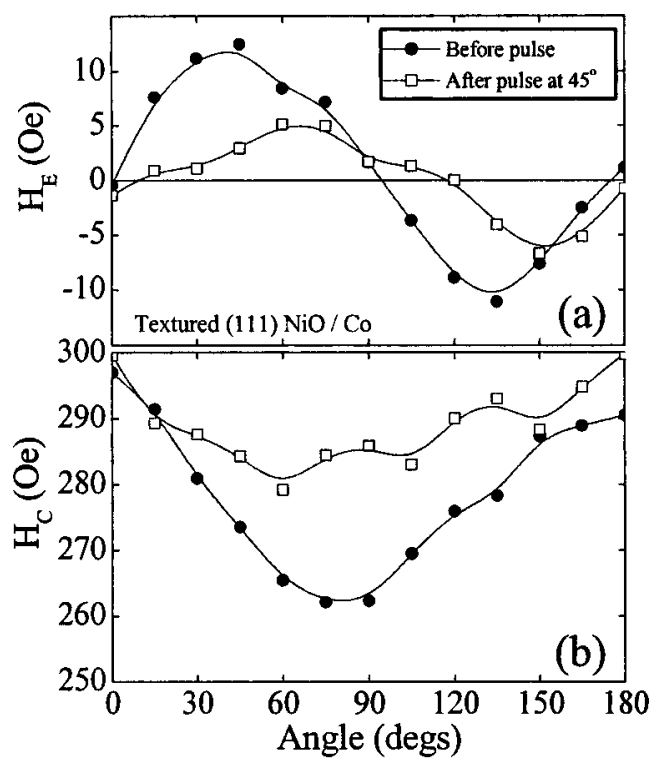

FIG. 3. Angular dependence of the exchange bias, $H_{E}$ (a), and coercivity, $H_{C}$ (b), for a $\mathrm{NiO} / \mathrm{Co}$ bilayer before $(-)$ and after the application of a pulse along $45^{\circ}(\square)$ away from the unidirectional anisotrony. Lines are guides for the eye. 
tals AFM domains have been found to reorient after being subjected to large fields, ${ }^{15}$ when in contact with a FM (Ref. 16) or when subjected to strains. ${ }^{17}$ Thus, if the AFM domains are modified by the pulse field, this effect should, in turn, influence $H_{E}$ and $H_{C}$ or even their angular dependence. Moreover, due to magnetostriction the pulsed fields could generate large strains at the interface, which could affect the AFM domain configuration. Note that since the AFM anisotropy for $\mathrm{NiO}$ is much smaller than for $\mathrm{FeMn}$, and in the latter case the unidirectional anisotropy is better established, one would expect larger domain based effects in the $\mathrm{NiO}$ case. Although the absolute change in $H_{E}$ is larger in FeMn, the fact that the angular dependence in textured $\mathrm{NiO}$ actually changes after the field pulse could be regarded as an indication of stronger reaction to the field pulse.

AFM materials have, below $T_{N}$, a transition from AFM to paramagnetic (PM) at high enough applied fields. ${ }^{18}$ Thus, in principle, in order to induce exchange bias it should be analogous to field cooling through $T_{N}$ or to apply large enough fields (larger than the AFM-PM phase transition) at a given temperature below $T_{N}$. In both cases, the AFM reaches a PM state and returns to the AFM state in the presence of a field. Since the samples are textured, i.e., without a clear AFM anisotropy axis in plane, pulses applied away from the original UAD should reset it to the pulse direction. However, the critical field for the AFM-PM transition, $H_{\mathrm{AFM}-\mathrm{PM}}$, is proportional to the exchange field of the AFM, which can be rather large in $\mathrm{FeMn}$ and $\mathrm{NiO}$ due to their large $T_{N} \cdot{ }^{18}$ For example, in bulk NiO, $H_{\mathrm{AFM}-\mathrm{PM}}>4000 \mathrm{kOe}$ at RT. ${ }^{19}$ Therefore, due to the limited strength of the field pulse, AFM-PM transitions would appear as an unlikely cause for the observed effects. However, AFM/FM bilayers typically exhibit rather large distributions of blocking temperatures, $\Delta T_{B},{ }^{20-22}$ with a sizable percentage of the AFM particles having $T_{B}$ close to RT. Moreover, considering that $H_{\mathrm{AFM}-\mathrm{PM}}$ is drastically reduced close to $T_{N}$ (or $T_{B}$ ) (Ref. 18) this would lead to some of the AFM particles to actually being sensitive to the field pulse. Consequently, $H_{E}$ and $H_{C}$ could be in practice partially reset. Due to the larger $\Delta T_{B}$ usually observed for $\mathrm{NiO},{ }^{20,21}$ this $\mathrm{AFM}$ would be expected to be more sensitive to the field pulses. Moreover, $\Delta T_{B}$ will lead to a "distribution of responses," which should result in an asymmetry of the hysteresis loop. ${ }^{23}$

Finally, it is well known that moderate fields applied along the easy axis of the AFM material induce a spin-flop transition $^{18}$ (e.g., for the NiO the spin-flop field can be estimated to be $\sim 90 \mathrm{kOe}$ at RT). ${ }^{19}$ This transition is known to affect exchange bias for highly crystalline AFM materials. ${ }^{6}$ Since the spin-flop transition is rather sensitive to the angle between the AFM easy axis and the applied field,$^{24}$ this effect should be expected to be small on textured AFMs (i.e., with a random orientation of AFM easy axes). However, due to random in-plane distribution of crystallites some of them would actually have their easy axes aligned within a few degrees of the pulse direction. Hence, a percentage of particles could contribute to the changes observed in the exchange bias properties and the loop asymmeties.

In conclusion, we have demonstrated that $H_{E}$ and $H_{C}$ can be controlled at room temperature (i.e., below $T_{N}$ ), after sample growth, by applying pulsed fields. The effects observed in textured $\mathrm{Fe}_{50} \mathrm{Mn}_{50}$ based bilayers are a decrease in $H_{E}$, while $H_{C}$ can either increase or decrease depending on the direction of the pulsed field. Similar effects are observed in textured $\mathrm{NiO}$ based bilayers, although in this case there is a reconfiguration of the unidirectional anisotropy axis towards the pulse direction. Hence, $\mathrm{NiO}$ appears to be more sensitive to the pulse, probably due to its smaller anisotropy or its larger $T_{B}$ distribution. These effects are probably due to field induced transitions in the AFM (AFM-PM or spinflop) or changes in the AFM domain structure.

This work was supported by CICYT (MAT2001-2555), DGR (2001SGR00189), the Generalitat de Catalunya (ITT2000-14), and Midi Pyrénées Council (CTP Grant). Two of the authors (J.S. and U.L.) thank the DGU and the Marie Curie Fellowship of the European Community program $\mathrm{Hu}-$ man Potential (HPMT-CT-2000-00106), respectively.

${ }^{1}$ J. Nogués and I. K. Schuller, J. Magn. Magn. Mater. 192, 203 (1999).

${ }^{2}$ B. Dieny, V. S. Speriosu, S. S. P. Parkin, B. A. Gurney, D. R. Wilhoit, and D. Mauri, Phys. Rev. B 43, 1297 (1991).

${ }^{3}$ M. G. Samant, J. A. Lüning, J. Stöhr, and S. S. P. Parkin, Appl. Phys. Lett. 76, 3097 (2000).

${ }^{4}$ T. Mewes, R. Lopusnik, J. Fassbender, B. Hillebrands, M. Jung, D. Engel, A. Ehresmann, and H. Schmoranzer, Appl. Phys. Lett. 76, 1057 (2000).

${ }^{5}$ J. Nogués, D. Lederman, T. J. Moran, and I. K. Schuller, Phys. Rev. Lett. 76, 4624 (1996); C. Leighton, J. Nogués, B. J. Jönsson-Åkerman, and I. K. Schuller, ibid. 84, 3466 (2000).

${ }^{6}$ J. Nogués, L. Morellon, C. Leighton, M. R. Ibarra, and I. K. Schuller, Phys. Rev. B 61, R6455 (2000).

${ }^{7}$ T. J. Moran and I. K. Schuller, J. Appl. Phys. 79, 5109 (1996); T. Ambrose and C. L. Chien, ibid. 83, 7222 (1998).

${ }^{8}$ N. J. Gökemeijer, J. W. Cai, and C. L. Chien, Phys. Rev. B 60, 3033 (1999); Y. F. Li, R. H. Yu, D. V. Dimitrov, and J. Q. Xiao, J. Appl. Phys. 86, 5692 (1999).

${ }^{9}$ P. Miltényi, M. Gierlings, M. Bamming, U. May, G. Güntherodt, J. Nogués, M. Gruyters, C. Leighton, and I. K. Schuller, Appl. Phys. Lett. 75, 2304 (1999).

${ }^{10}$ P. A. A. van der Heijden, T. F. M. M. Maas, W. J. M. de Jonge, J. C. S. Kools, F. Roozeboom, and P. J. van der Zaag, Appl. Phys. Lett. 72, 492 (1998); T. Hughes, K. O'Grady, H. Laidler, and R. W. Chantrell, J. Magn Magn. Mater 235, 329 (2001).

${ }^{11}$ F. Nolting et al., Nature (London) 405, 767 (2000).

${ }^{12}$ C. Leighton, A. Hoffmann, M. R. Fitzsimmons, J. Nogués, and I. K. Schuller, Philos. Mag. B 81, 1927 (2001).

${ }^{13}$ A. P. Malozemoff, Phys. Rev. B 35, 3679 (1987); P. Miltényi, M. Gierlings, J. Keller, B. Beschoten, G. Güntherodt, U. Nowak, and K. D. Usadel, Phys. Rev. Lett. 84, 4224 (2000).

${ }^{14}$ A. Hochstrat, C. Binek, and W. Kleemann, Phys. Rev. B 66, 092409 (2002).

${ }^{15}$ S. Saito, M. Miura, and K. Kurosawa, J. Phys. C 13, 1513 (1980).

${ }^{16}$ H. Ohldag, A. Scholl, F. Nolting, S. Anders, F. U. Hillebrecht, and J. Stöhr, Phys. Rev. Lett. 86, 2878 (2001).

${ }^{17}$ J. C. Marmeggi and J. Baruchel, J. Magn. Magn. Mater. 10, 14 (1979).

${ }^{18} \mathrm{~K}$. Yoshida, Theory of Magnetism, Springer Series in Solid State Scineces, Vol. 122 (Springer, Berlin, 1991), Chap. 6.

${ }^{19}$ Values estimated according to Ref. 18 from the data given in M. T. Hutchings and E. J. Samuelsen, Phys. Rev. B 6, 3447 (1972).

${ }^{20}$ S. Soeya, T. Imagawa, K. Mitsuoka, and S. Narishige, J. Appl. Phys. 76, 5356 (1994)

${ }^{21}$ J. Fujikata, K. Hayashi, H. Yamamoto, and M. Nakada, J. Appl. Phys. 83, 7210 (1998).

${ }^{22}$ J. P. Nozières, S. Jaren, Y. B. Zhang, A. Zeltser, K. Pentek, and V. S. Speriosu, J. Appl. Phys. 87, 3920 (2000).

${ }^{23}$ Asymmetric loops are common in exchange bias systems, e.g., M. R. Fitzsimmons, P. Yashar, C. Leighton, I. K. Schuller, J. Nogués, C. F. Majkrzak, and J. A. Dura, Phys. Rev. Lett. 84, 3986 (2000). However, in our case there is an obvious asymmetry only after the field pulse.

${ }^{24}$ G. P. Felcher and R. Kleb, Europhys. Lett. 36, 455 (1996). 